\title{
Linear and nonlinear Fano resonance on two-dimensional magnetic metamaterials
}

\author{
H. Liu, ${ }^{1,2}$ G. X. Li, ${ }^{1, *}$ K. F. Li, ${ }^{1}$ S. M. Chen, ${ }^{1}$ S. N. Zhu, ${ }^{2}$ C. T. Chan, ${ }^{3}$ and K. W. Cheah ${ }^{1}$ \\ ${ }^{1}$ Department of Physics, Hong Kong Baptist University, Hong Kong, China \\ ${ }^{2}$ Department of Physics, National Laboratory of Solid State Microstructures, Nanjing University, Nanjing 210093, China \\ ${ }^{3}$ Department of Physics and Nano Science and Technology Program, Hong Kong University of Science and Technology,
} Clearwater Bay, Hong Kong, China

(Received 19 September 2011; revised manuscript received 20 November 2011; published 22 December 2011)

\begin{abstract}
We demonstrate that both linear and nonlinear Fano resonances can be realized on two dimensional magnetic metamaterials. The Fano resonance comes from the interference between localized magnetic plasmon resonance and propagating surface plasmon polaritons. When studying the linear optical response of the metamaterial structure, this interference phenomenon was observed in the ellipsometric spectrum. By finely tailoring the geometrical parameters of the magnetic metamaterial device, the nonlinear Fano response was tuned to a nearinfrared wavelength (1.61-1.8 $\mu \mathrm{m})$ of femtosecond pump laser, and Fano-type modulation of the third harmonic generation was found and agrees well with our theoretical model.
\end{abstract}

DOI: 10.1103/PhysRevB.84.235437

PACS number(s): 78.67.Bf, 73.20.Mf, 42.65.Ky, 42.70.Qs

\section{INTRODUCTION}

Recent studies on nonlinear optical plasmonic nanostructures show that the efficiency of nonlinear optical processes can be efficiently enhanced by strongly localized plasmonic excitations. ${ }^{1-16}$ By using enhanced optical nonlinearity in metallic nanostructures, plasmonic/metamaterial devices have been used for ultrafast optical switching applications. ${ }^{17-19}$ In the field of nonlinear optics, while second-order processes have been extensively studied on various plasmonic/metamaterial nanostructures, ${ }^{2-10}$ third-order nonlinear optical phenomena on metallic nanostructures are attracting more attention. For example, surface enhanced four-wave mixing on gold plasmonic nanostructures ${ }^{11,12,14}$ and a plasmon-enhanced third harmonic generation ${ }^{15,16}$ were demonstrated by virtue of a light confinement effect of plasmonic excitations. Although the strong field localization discussed usually refers to the electric field, the magnetic field also significantly contributes to the enhancement of the electric field under resonant conditions. In the pioneering work of Pendry et al. ${ }^{20}$ magnetic resonanceinduced field localization was proposed to enhance the efficiency of nonlinear optical processes. By using the magnetic plasmon resonance (MPR) mode, the energy of incident light is absorbed into an equivalent inductance and capacitance (LC) circuit and strongly localized in an ultra-small volume of the equivalent capacitor. This strong field localization makes MPR nanostructure a good candidate for surface-enhanced nonlinear optical processes. Based on this mechanism, enhanced nonlinear optical processes in magnetic metamaterials, i.e. third harmonic generation (THG), ${ }^{21}$ nanolasers, ${ }^{22,23}$ and spasers, ${ }^{24,25}$ have been reported in the past several years.

In complex plasmonic systems, usually two or more resonant modes exist. The interference between these plasmonic modes can result in strong Fano resonance. ${ }^{25}$ Linear optical properties of Fano resonance in plasmonic nanostructures have been extensively studied recently. ${ }^{26-33}$ Integration of Fano resonance into nonlinear optics is expected to offer more optical functionalities, for example, enhanced nonlinear optical response of the free carriers in metamaterial/carbon nanotube composites ${ }^{30}$ and fishnet metamaterials ${ }^{34,35}$ have been utilized to fabricate ultrafast optical switches.
In this work, we demonstrate that the third harmonic generation, one kind of radiative nonlinear optical process, can be spectrally modulated using Fano-resonant magnetic metamaterials. The device is a trilayer nanostructure, consisting of a gold slab, indium tin oxide (ITO), and a two-dimensional gold nanodisc. This Fano-resonant metamaterial supports both the localized MPR mode and propagating surface plasmon polaritons (SPP). By finely tuning the interference between localized MPR and propagating SPP modes at a near-infrared wavelength, the surface polarization of a pump laser on a metamaterial induces Fano-resonant modulation. Spectrally resolved third harmonic generation on this metal/dielectric hybrid nanostructure was demonstrated to show a Fanoresonant response. This indicates that third-order and other nonlinear optical processes can be tailored by engineering the coupling of MPR and SPP modes. The interference between these two kind of modes provides us a more flexible way to design nonlinear optical nanodevices.

\section{DEVICE FABRICATION}

The cross section of a nanostructure is shown in Fig. 1(a), and a unit cell is composed of a gold nanodisc fabricated on top of an ITO/gold slab. Two samples (A and B) were fabricated using the e-beam lithography, thin film deposition, and metal lift-off process. The scanning electron microscope (SEM) pictures of the two samples are shown in Figs. 1(b) and 1(c). Thicknesses of the gold nanodisc, ITO, and gold slab are $50 \mathrm{~nm}$ thick, respectively. The disc diameter of A is $320 \mathrm{~nm}$ with a period of $600 \mathrm{~nm}$, while for sample B, the diameter of the disc is $360 \mathrm{~nm}$ with the period of $910 \mathrm{~nm}$.

\section{OPTICAL CHARACTERIZATION}

Linear optical properties of this metamaterial were characterized using a Fourier transform infrared (FTIR) spectrometer and a spectroscopic ellipsometer. In the FTIR experiment, the reflection spectra of unpolarized light [shown in Fig. 2(a)] on sample $\mathrm{A}$ and $\mathrm{B}$ were measured under normal incidence. A thick $(100 \mathrm{~nm})$ gold film-coated silicon substrate was used as a reference cell. The relative reflection spectrum is calculated 


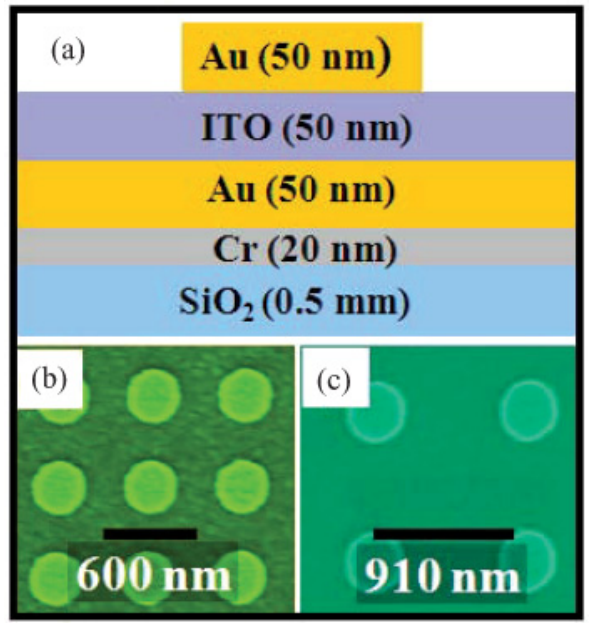

FIG. 1. (Color online) (a) Cross section of a metamaterial device: gold circular disc/ITO layer/gold slab; (b) and (c) SEM picture of MPC samples: gold disc array/ITO layer/gold slab/Cr layer/quartz. Thickness of gold/ITO/gold/Cr is $50 \mathrm{~nm} / 50 \mathrm{~nm} / 50 \mathrm{~nm} / 30 \mathrm{~nm}$. (b) Sample A (period: $600 \mathrm{~nm}$ and disc diameter $320 \mathrm{~nm}$ ); (c) Sample B (period: $910 \mathrm{~nm}$ and disc diameter $360 \mathrm{~nm}$ ).

from the ratio of reflected intensity on the nanostructure and gold reference cell. The magnetic resonant dips were observed at $1.51 \mu \mathrm{m}$ for sample A (line with triangle symbol) and $1.62 \mu \mathrm{m}$ for sample B (line with circle symbol). At the
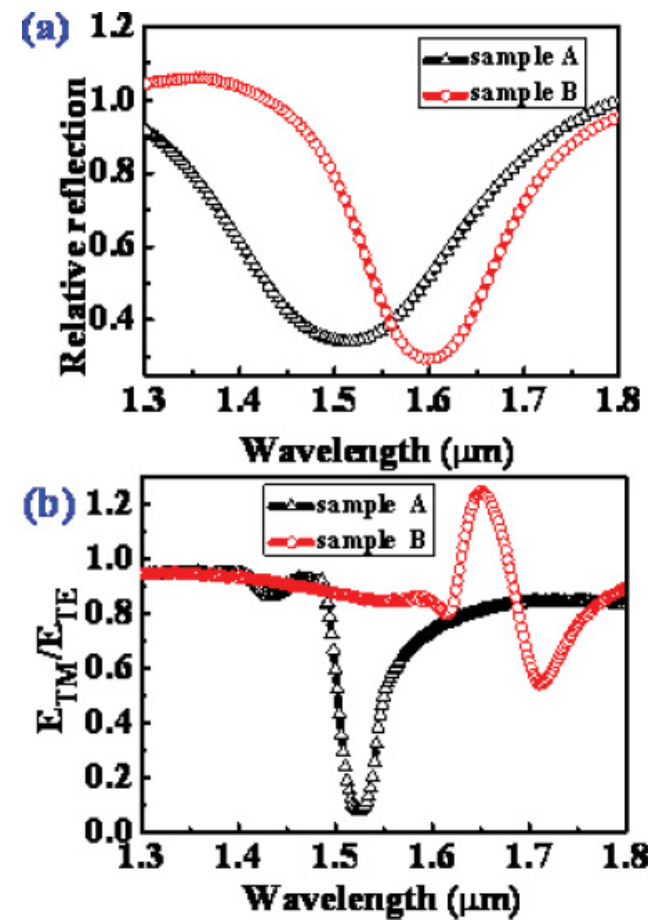

FIG. 2. (Color online) Relative reflection spectra on two samples, reference sample: gold (100 nm thick)/silicon. (a) Reflection spectra measured using FTIR under normal incidence condition $\left(\theta=0^{\circ}\right)$, sample A (line with triangle symbol) and B (line with circle symbol). (b) Reflection ratio of $E_{\mathrm{TM}} / E_{\mathrm{TE}}$ on sample A (line with triangle symbol) and sample B (line with circle symbol) are measured using spectroscopic ellipsometer at incident angle of $\left(\theta=52^{\circ}\right)$.

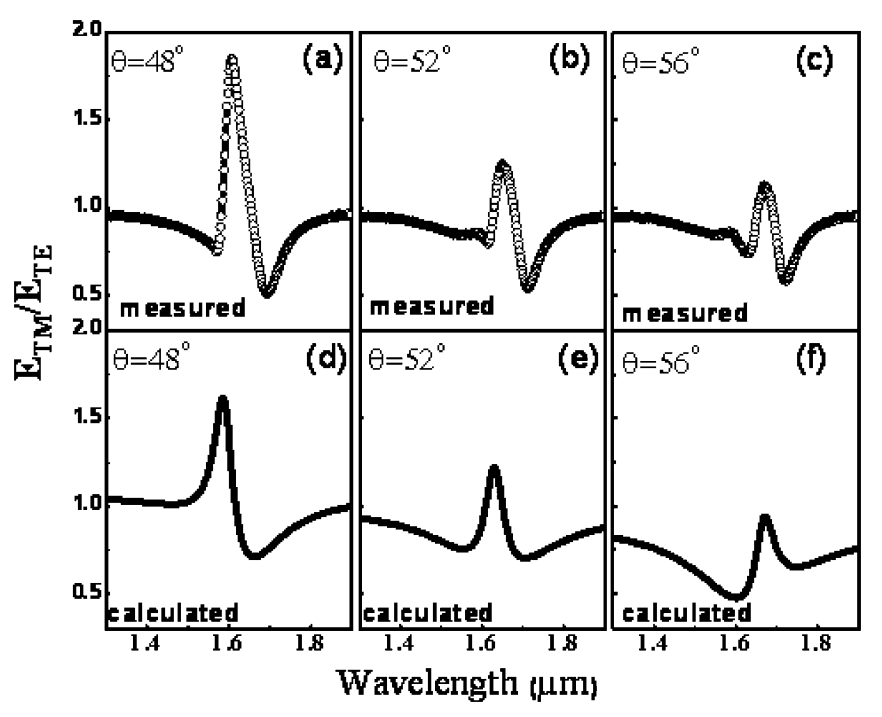

FIG. 3. Reflection ratio of $E_{\mathrm{TM}} / E_{\mathrm{TE}}$ on sample B under different incident angle $\left(\theta=48^{\circ}, 52^{\circ}, 56^{\circ}\right)$ : (a)-(c) experimentally measured results (dashed line); (d)-(f) theoretically calculated results (solid line).

resonant wavelength, the induced currents in the disc and slab are antiparallel, which corresponds with the MPR mode, as reported before. ${ }^{23,36,37}$ In the ellipsometry measurement, the reflection coefficient ratio of transverse magnetic (TM) and transverse electric (TE) waves $\eta=E_{\mathrm{TM}} / E_{\mathrm{TE}}$ was retrieved using the rotating polarizer technique. In Fig. 2(b), spectrally resolved $\eta$ is measured at the incident angle: $\theta=52^{\circ}$. For sample A, pure MRR mode still exists and has a little red shift compared to the FTIR result [Fig. 2(a)]. However, for sample B, a Fano-type optical response at the MPR wavelength is observed. This interesting phenomenon means that the resonance in sample B is not a pure MPR mode. Angle-resolved measurement (incident angle $\theta$ was tuned from $48^{\circ}$ to $56^{\circ}$ ) was then used to study the mechanism behind this phenomenon. For sample A, magnetic resonance mode is not sensitive to incident angles, and the reflection dip almost remains at the same wavelength, as shown in Fig. 2(a). In comparison, angle-resolved reflection ellipticity of sample B [Figs. 3(a)-3(c)] shows that the Fano-resonant dip is very sensitive to the incident angle and shifts to a longer wavelength when increasing the incident angle.

\section{THEORETICAL ANALYSIS OF FANO RESONANCE}

As MPR is one kind of localized mode with a definite resonant frequency, it should not be sensitive to incident angles. ${ }^{36}$ From our previous study on plasmonic ellipsometry, ${ }^{38}$ the angle-dependent shift of Fano resonance on sample B is related to the excitation of propagating surface plasmon polaritons. The excitation of propagating surface plasmon is governed by the following momentum conservation condition: $k_{\|}+k_{\mathrm{spp}}^{\prime}-G^{\prime}=0$ (Fig. 4), ${ }^{39}$ where, $k_{\|}=(\omega / c) \sin \theta$ is the $x$ component of the vacuum wave vector; $k_{\mathrm{spp}}=k_{\mathrm{spp}}^{\prime}+i k_{\mathrm{spp}}^{\prime \prime}$ is the wave vector of SPP waves, with the imaginary part $k_{\mathrm{spp}}^{\prime \prime}$ representing the ohmic loss of the SPP wave; $G=2 \pi /$ period $+i G^{\prime \prime}$ is the first order of the reciprocal vector, and $G^{\prime \prime}$ is the radiative loss. For sample B (with period of $910 \mathrm{~nm}$ ), the 


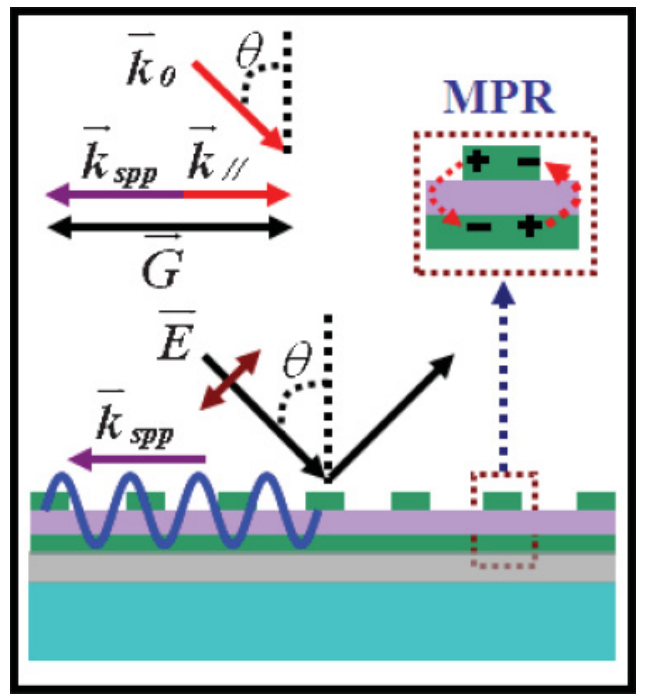

FIG. 4. (Color online) Both localized MPR mode and propagating SPP mode are excited by the TM wave. Here, $\vec{G}=2 \pi /$ period is the reciprocal vector.

wavelength of first-order SPP is $\sim 1.63 \mu \mathrm{m}$ at the incident angle: $\theta=52^{\circ}$, which is very close to the MPR mode with a wavelength of $\sim 1.62 \mu \mathrm{m}$. Thus, both SPP and MPR modes can be excited (see Fig. 4), and the interference between the MPR and SPP modes shows a Fano-type shape in the reflection spectrum (Fig. 3). But for sample A, as its period $(600 \mathrm{~nm})$ is too small, the first-order SPP $\left(\sim 1.08 \mu \mathrm{m}\right.$ at: $\left.\theta=52^{\circ}\right)$ is far away from the MPR wavelength $(1.51 \mu \mathrm{m})$; that is why there is no similar Fano-type resonance.

Once propagating surface plasmon polaritons are excited, the reflected TM wave shows a resonant dip at the plasmon resonant frequency, while the reflected TE wave just shows a slowly varying response. At the resonant wavelength of the surface plasmon polaritons, the TM polarized reflection spectra for SPP is defined as: ${ }^{39}$

$$
r_{\mathrm{spp}}=r_{p 0}\left(1-\frac{2 i G^{\prime \prime}}{k_{\|}+k_{\mathrm{spp}}-G}\right) .
$$

Here, $r_{p 0}=\left(n_{\text {gold }} \cos \theta-\cos \theta_{\text {gold }}\right) /\left(n_{\text {gold }} \cos \theta+\cos \right.$ $\left.\theta_{\text {gold }}\right)$ is the reflection coefficient on the air/gold surface $\left(\theta_{\text {gold }}\right.$ is the refractive angle in gold film); $G_{0}=(2 \pi / p)(1+i \Gamma)$ $=G^{\prime \prime}+i G^{\prime \prime}$ is the reciprocal vector and $\Gamma$ is the radiative damping rate of surface plasmon polaritons [estimated from Fig. 2(b)]; $k_{\text {spp }}(\omega)=k_{0}(\omega) \sqrt{\varepsilon_{\text {gold }} \varepsilon_{\text {air }} /\left(\varepsilon_{\text {gold }}+\varepsilon_{\text {air }}\right)}$ is the wave vector of the SPP wave at interface between gold and air.

As was reported before, ${ }^{37}$ the nanostructure in Fig. 1(a) is regarded as an equivalent LC circuit and described by Lagrangian as $L=\frac{L}{2} \dot{Q}^{2}-\frac{1}{2 C} Q^{2}$. Here, $L$ and $C$ are inductance and capacitance of the LC circuit, $Q$ is the net charge in the capacitor, and $\dot{Q}$ is the induced current in the inductance. In the presence of ohmic dissipation and an external driving field, the Euler-Lagrange equation can be written as: $\frac{d}{d t}\left(\frac{\partial L}{\partial \dot{Q}}\right)-\frac{\partial L}{\partial Q}=-\frac{\partial R}{\partial \dot{Q}}+$ e.m.f., where $R=R_{\text {eff }} \dot{Q}^{2} / 2\left(R_{\text {eff }}\right.$ is the effective resistance of the nanostructure) and e.m.f. (for normal incident $B_{0}$ field $)$ is given by: e.m.f. $=-\frac{d B_{0}}{d t} S_{\text {eff }}\left(S_{\text {eff }}\right.$ is the effective cross-section area between patch and slab). If we define an effective magnetic dipole as: $m=S_{\text {eff }} \dot{Q}=\alpha_{m} B_{0}$ $\left(\alpha_{m}\right.$ is the effective magnetic polarizability of the magnetic resonator), the Euler-Lagrange equation has following solution: $\alpha_{m}=\frac{S_{\mathrm{eff}}^{2}}{L} \frac{\omega^{2}}{\left(\omega^{2}-\omega_{0}^{2}\right)+i \gamma \omega}$. At the MPR frequency $\left[\omega_{0}\right.$ is estimated from Fig. 2(b)], electromagnetic energy is absorbed by magnetic resonators and finally converted to heat loss. For a pure MPR mode, a Lorenz-shape absorption dip will be observed in the reflected spectrum. Based on the above Lagrangian model, the reflection coefficient of MRC can be described by $r_{\mathrm{MPR}}=\left(1-C_{\mathrm{MPR}}\right)$, where the absorption coefficient is $C_{\mathrm{MPR}}=f_{\mathrm{MPR}} \frac{i \gamma_{m} \omega}{\left(\omega^{2}-\omega_{0}^{2}\right)+i \gamma_{m} \omega},{ }^{40}$ and $f_{\mathrm{MPR}}$ is the light coupling efficiency of magnetic resonators. For TM polarization, as the magnetic field is always perpendicular to the incident plane, the electromotive force is not sensitive to the incident angle, so the reflection coefficient can be given by $r_{\mathrm{MPR}-\mathrm{TM}}=r_{p 0}\left(1-C_{\mathrm{MPR}}\right)$. In comparison, $r_{\mathrm{MPR}-\mathrm{TE}}=r_{s 0}(1$ $-C_{\mathrm{MPR}} \cos \theta$ ) is used to calculate the reflection coefficient for TE polarization, as electromotive force is dependent on the incident angle $\theta$ as: e.m.f. $=-\frac{d B_{0}}{d t} S_{\text {eff }} \cos (\theta)$ in this case.

For the TM wave, the total reflection coefficient is given by the interference between the SPP and MPR modes:

$$
r_{\mathrm{TM}}=\left[f_{\mathrm{spp}} r_{\mathrm{spp}}+\left(1-f_{\mathrm{spp}}\right) r_{\mathrm{MPR} \_\mathrm{TM}}\right],
$$

where $f_{\text {spp }}$ and $\left(1-f_{\text {spp }}\right)$ are the weight factors to describe the ratio of the SPP and TM-polarized MPP modes, respectively. For the TE wave, the modulated reflection spectrum is mainly due to the absorption of the TE-polarized MPR mode, the approximated reflection coefficient is:

$$
r_{\mathrm{TE}}=r_{\mathrm{MPR} \_\mathrm{TE}} .
$$

To compare theoretical calculation with experimental result, a modification factor $f_{\text {ellip }}=1.583$ is introduced. As is shown in Figs. 3(d)-3(f) (solid line), the theoretical ratio of reflection coefficients $\left(\eta=f_{\mathrm{ellip}} r_{\mathrm{TM}} / r_{\mathrm{TE}}\right)$ for TM and TE polarization is shown $\left(f_{\mathrm{spp}}=0.16\right.$ and $f_{\mathrm{MPR}}=1.1$ are chosen). It can be found that Fano resonances appear in the calculated results and agree with experimental observations [Figs. 3(a)-3(c)].

\section{NONLINEAR OPTICAL EXPERIMENT}

Nonlinear optical properties of Fano-resonant metamaterial were studied using the third harmonic generation process. A femtosecond laser pumped optical parametric amplifier (OPA, TOPAS-C) system was used in this experiment. The output wavelength of OPA can be tuned from 1.61 to $1.8 \mu \mathrm{m}$ with a repetition frequency of $1 \mathrm{kHz}$ and pulse duration of $\sim 100$ femtosecond. As is shown in Fig. 5, the horizontally polarized (electric field parallel to the incident plane) pump laser was incident at an angle of $52^{\circ}$. After being focused by a lens with a focal length of $150 \mathrm{~mm}$, the near-infrared laser pulse was incident onto the metamaterial device. The laser spot size is about 120 by $100 \mu \mathrm{m}$, which is less than the total area of the nanostructure.

Radiation direction of the third harmonic generation is determined by the momentum conservation condition, which is given by following equation: ${ }^{9}$

$$
k_{0}(3 \omega) \sin (\phi)=3 k_{0}(\omega) \sin (\theta)+m \frac{2 \pi}{p_{x}}+n \frac{2 \pi}{p_{y}}
$$




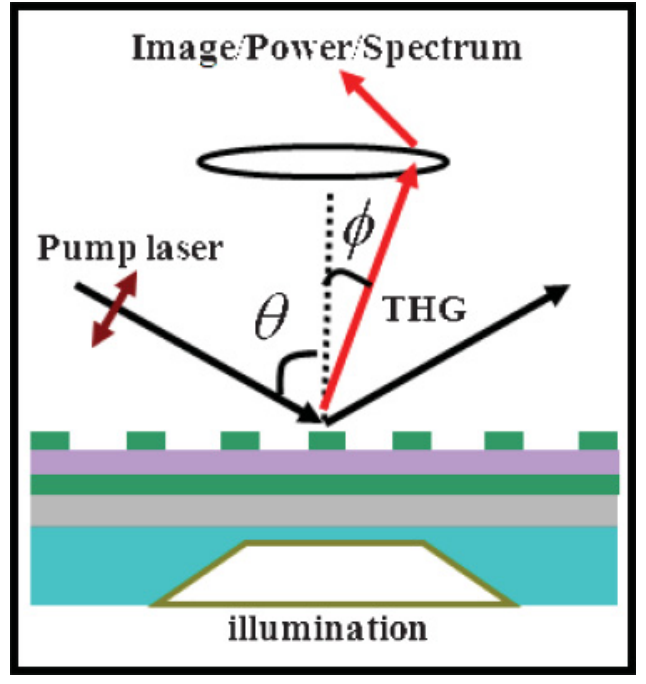

FIG. 5. (Color online) Experimental setup of the third harmonic generation. Near infrared was incident on the sample at angle $(\theta=$ $52^{\circ}$ ). The surface emission of THG (in-plane first order) was focused and then was imaged by CCD camera. The spectrum and intensity of the third harmonic generation were recorded by an Ocean Optics spectrometer and a Newport power detector.

where $\phi$ is the radiation angle of the third harmonic generation to the surface normal of the nanostructure, $m$ and $n$ represent diffraction orders. For the in-plane first order $(m=-1, n=0)$, radiation angle $(\phi)$ of THG ranges from $11.4^{\circ}$ to $7.39^{\circ}$, while the excitation wavelength is tuned from 1610 to $1800 \mathrm{~nm}$. The THG signal was collected by silicon-based charge coupled device (CCD) detector and spectrometer (Ocean Optics 4000) after filtering the fundamental wave. The output power was measured by a photodiode (Newport 818-UV). Two linear polarizers were used to control the power and polarization of the pump laser; a Newport power detector (818-IR) was used to monitor the power variation of the pump laser (at near near-infrared wavelength) from the side beam of the beam splitter.

An imaging system was set up to monitor the position of the nanostructure and pump laser using a Xenon lamp for illumination light. In the bright-field mode [Figs. 6(a) and 6(b)], it can be found that both the radiation spot (marked by dashed line) of the third harmonic generation and the nanostructure can be imaged by CCD camera. After turning off the illumination light, dark-field imaging of the third harmonic generation [Figs. 6(c) and 6(d)] was realized. The spectrum of the third harmonic generation was measured using an Ocean Optics spectrometer after filtering the pump laser. In Fig. 7, THG spectra on sample A, where the bandwidth of the THG peak is about $9 \mathrm{~nm}$, are measured using fundamental wavelengths of 1620 and $1640 \mathrm{~nm}$. The relative efficiency of THG (the intensity ratio of THG and the pump laser) on samples A and B is given in Fig. 8. For sample A, for which the resonant wavelength is at $\sim 1.51 \mu \mathrm{m}$, the third harmonic generation efficiency decreases when the pump wavelength is tuned from 1.61 to $1.8 \mu \mathrm{m}$ [Fig. 8(a)]. However, the third harmonic generation on sample B is quite different, in which a Fano-resonant modulation was found [Fig. 8(b)].

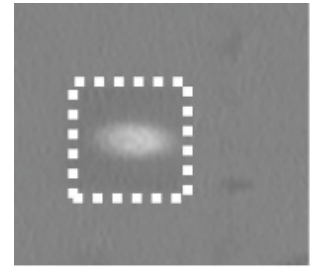

(a) $1740 \mathrm{~nm}$

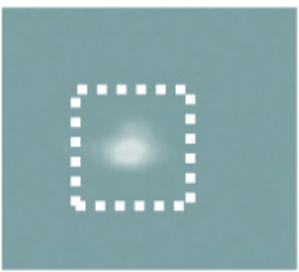

(c) $1650 \mathrm{~nm}$

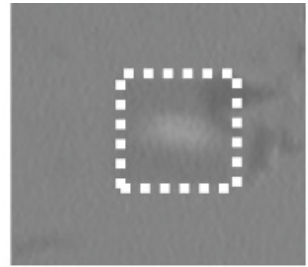

(b) $1800 \mathrm{~nm}$

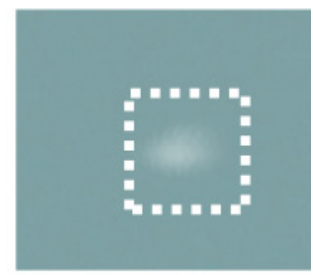

(d) $1790 \mathrm{~nm}$
FIG. 6. (Color online) (a) and (b) Bright-field and (c) and (d) dark field imaging of the third harmonic generation on sample A. Fundamental wavelength is: (a) $1740 \mathrm{~nm}$, (b) $1800 \mathrm{~nm}$, (c) $1650 \mathrm{~nm}$, and (d) $1790 \mathrm{~nm}$, respectively.

To elucidate the Fano-modulated third harmonic generation on metamaterial devices, a nonlinear modulation factor of THG is defined: $\eta_{\mathrm{THG}}=\eta_{0}+\eta_{\mathrm{MPC}}$, where $\eta_{0}$ and $\eta_{\mathrm{MPC}}$ come from the intrinsic and field enhancement contributions. The surface nonlinear polarization on the metamaterial device can be written as: $\vec{P}_{\mathrm{SNL}}^{(3)}=\chi^{(3)}:\left[\eta_{\mathrm{THG}} \vec{E}_{0}(\omega)\right]^{3}$, and $\vec{E}_{0}$ is the electric field of the pump laser. ${ }^{41}$ The intrinsic THG contribution $\left(\eta_{0}\right)$ in this experiment is attributed to $\chi^{(3)}$ of gold and ITO. As reported in previous work, ${ }^{15}$ the gold plasmonic nanostructure shows highly efficient THG radiation at nearinfrared wavelengths $(1.61$ to $1.8 \mu \mathrm{m})$. Most interestingly is that the three pumping photons in the THG process do not induce interband transition (from $5 d$ to $6 s p$ ) in gold, as the band gap of $5 d-6 s p$ for gold is about $2.4 \mathrm{eV}(\sim 520 \mathrm{~nm})$. The excited electrons in gold are just pumped to a virtual state near the Fermi level; the dephasing time of electrons at this state should be an ultrafast process, and this explains the efficient THG radiation at this optical regime. In addition, the third-order susceptibility of ITO should also be taken into

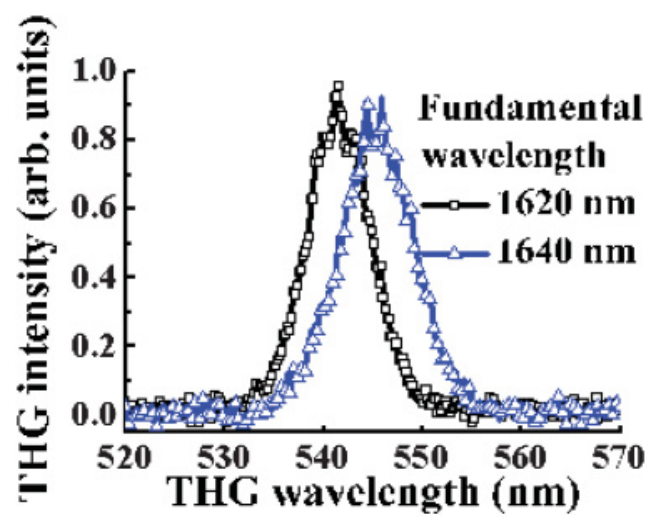

FIG. 7. (Color online) Spectra of the third harmonic generation on sample A, fundamental wavelength: $1620 \mathrm{~nm}$ (line with square symbol); $1640 \mathrm{~nm}$ (line with triangle symbol). 


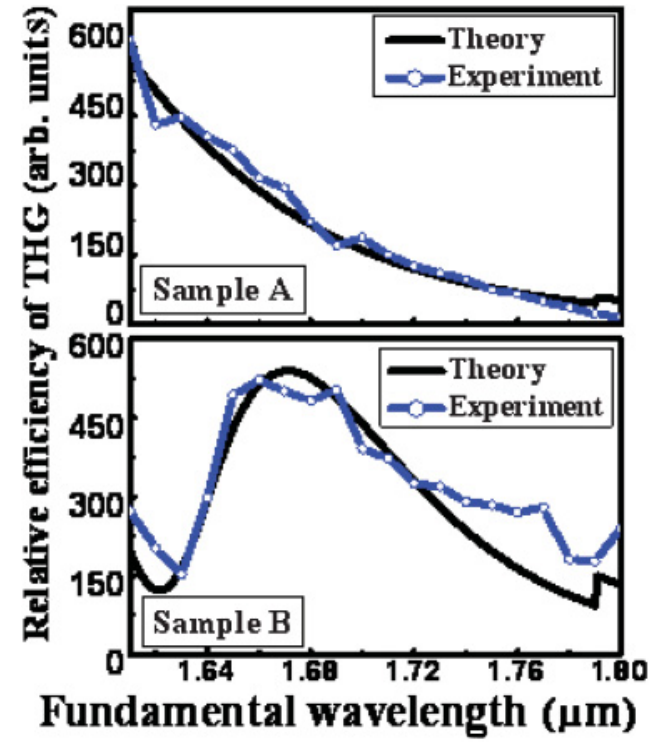

FIG. 8. (Color online) Spectrally resolved THG on samples A and $\mathrm{B}$ at an incident angle of $\left(\theta=52^{\circ}\right)$. (a) Experimental (line with circle symbol) and calculated (solid line) THG efficiency of sample A. (b) Experimental (line with circle symbol) and calculated THG efficiency of sample B (solid line).

account, ${ }^{42}$ especially for magnetic resonant metamaterial, in which the energy of the pump laser is also strongly localized in the ITO spacer layer.

For sample A, field localization mainly comes from the magnetic plasmon resonance; the modulation factor of the electric field can be described by: $\eta_{\mathrm{MPC}}=Q_{\mathrm{MPR} \_\mathrm{A}}\left|C_{\mathrm{MPR} \_\mathrm{A}}\right|$. However, for sample B, $\eta_{\text {MPC }}$ comes from the contribution of interference between the MPR and SPP modes, and it can be written as: $\eta_{\mathrm{MPC}}=\mid Q_{\mathrm{MPR} \_\mathrm{B}} C_{\mathrm{MPR} \_\mathrm{B}}+Q_{\mathrm{spp}} C_{\mathrm{spp}} \mathrm{l}$, where $Q_{\mathrm{MPR}}$ and $Q_{\text {spp }}$ are the weight factors of the MPR and SPP modes (ratio $=7$ is chosen in the calculation). Assuming that ITO and gold have similar third-order susceptibility, ${ }^{42}$ radiation intensity of THG has a cubic relation with respect to the power of the pump laser; thus, we have $I_{\mathrm{THG}} \sim\left|\vec{P}_{\mathrm{SNL}}\right|^{2} \sim\left|\eta_{\mathrm{MPC}}^{2} \vec{E}_{0}^{2}\right|^{3}$. The calculated results (line with triangle symbol) agree with measured ones (line with circle symbol). Based on the above theoretical analysis, we know that the Fano-resonant THG comes from the modulated-surface nonlinear polarization of the pumped electric field, indicating that nonlinear polarizations from surface plasmon polaritons and MPR modes have an interference effect. By tuning the resonant frequency and phase delay between the SPP and MPR modes, the plasmonic excitation around Fano-resonant wavelengths can be switched from the localized magnetic resonant mode to the propagating surface plasmon mode or vice versa.

\section{CONCLUSIONS}

In conclusion, we designed a two-dimensional magnetic metamaterial. Both localized MPR and propagating SPP modes can be excited in such a system. Through the interference between the MPR and SPP excitations, linear Fano effect is found from ellipsometric spectra. The nonlinear optical process on metamaterial devices was studied through the THG process. By modulating nonlinear polarization through the interference between the MPR and SPP modes, Fano-resonant THG was for the first time observed on a magnetic metamaterial device. The integration of Fano resonance into a nonlinear optical MPR device will provides many more opportunities for realizing functional optical nanodevices. Although we only studied THG here, such a nonlinear Fano-resonant effect can also be applied to other nonlinear optical processes, such as high harmonic generation, surface enhanced Raman, and four-wave mixing, etc.

\section{ACKNOWLEDGMENTS}

G.X. would like to thank Nelson $\mathrm{Li}$ and J. N. Wang for their support on device fabrication. Device fabrication was supported by University Grant Council with Grant SEG_HKUST10. H.L. was financially supported by K. C. Wong Education Foundation, National Natural Science Foundation of China (Nos. 11021403, 11074119, 10874081, 60990320, and 11004102), and by the National Key Projects for Basic Researches of China (Nos. 2010CB630703, 2012CB921501, and 2012CB933501). G.X. and K.W. would like to thank John Pendry, T. Li, and S. M. Wang for fruitful discussions.
*Corresponding author: Department of Physics, Hong Kong Baptist University, Kowloon Tong, Honk Kong; sunview1981@hotmail.com

${ }^{1}$ S. Kujala, B. K. Canfield, M. Kauranen, Y. Svirko, and J. Turunen, Phys. Rev. Lett. 98, 167403 (2007).

${ }^{2}$ J. Butet, J. Duboisset, G. Bachelier, I. Russier-Antoine, E. Benichou, C. Jonin, and P. F. Brevet, Nano Lett. 10, 1717 (2010).

${ }^{3}$ J. Butet, G. Bachelier, J. Duboisset, F. Bertorelle, I. RussierAntoine, C. Jonin, E. Benichou, and P. F. Brevet, Opt. Express 18, 22314 (2010).

${ }^{4}$ J. Butet, G. Bachelier, I. Russier-Antoine, C. Jonin, E. Benichou, and P. F. Brevet, Phys. Rev. Lett. 105, 077401 (2010).

${ }^{5}$ Y. Zeng, W. Hoyer, J. Liu, S. W. Koch, and J. V. Moloney, Phys. Rev. B 79, 235109 (2009).
${ }^{6}$ C. G. Biris and N. C. Panoiu, Phys. Rev. B 81, 195102 (2010).

${ }^{7}$ V. K. Valev, A. V. Silhanek, N. Smisdom, B. De Clercq, W. Gillijns, O. A. Akstipetrov, M. Ameloot, V. V. Moshchalkov, and T. Verbiest, Opt. Express 18, 8286 (2010).

${ }^{8}$ F. X. Wang, F. J. Rodriguez, W. M. Albers, R. Ahorinta, J. E. Sipe, and M. Kauranen, Phys. Rev. B 80, 233402 (2009).

${ }^{9}$ N. J. Borys, M. J. Walter, and J. M. Lupton, Phys. Rev. B 80, 161407(R) (2009).

${ }^{10}$ A. Nahata, R. A. Linke, T. Ishi, and K. Ohashi, Opt. Lett. 28, 423 (2003).

${ }^{11}$ J. Renger, R. Quidant, N. van Hulst, and L. Novotny, Phys. Rev. Lett. 104, 046803 (2010).

${ }^{12} \mathrm{P}$. Genevet, J. P. Tetienne, E. Gatzogiannis, R. Blanchard, M. A. Kats, M. O. Scully, and F. Capasso, Nano Lett. 10, 4880 (2010). 
${ }^{13}$ K. D. Ko, A. Kumar, K. H. Fung, R. Ambeka, G. L. Liu, N. X. Fang, and K. C. Toussaint, Nano Lett. 11, 61 (2011).

${ }^{14}$ V. K. Valev, A. V. Silhanek, N. Verellen, W. Gillijns, P. Van Dorpe, O. A. Aktsipetrov, G. A. E. Vandenbosch, V. V. Moshchalkov, and T. Verbiest, Phys. Rev. Lett. 104, 127401 (2010).

${ }^{15}$ G. X. Li, T. Li, H. Liu, K. F. Li, S. M. Wang, S. N. Zhu, and K. W. Cheah, Appl. Phys. Lett. 98, 261909 (2011).

${ }^{16}$ T. Xu, X. Jiao, and S. Blair, Opt. Express 17, 23582 (2009).

${ }^{17}$ K. F. Macdonald, Z. L. Samson, M. I. Stockman, and N. I. Zheludev, Nat. Photon. 3, 55 (2008).

${ }^{18}$ D. Pacific, H. J. Lerec, and H. A. Atwater, Nat. Photon. 1, 402 (2007).

${ }^{19}$ G. A. Wurtz, R. Pollard, W. Hendren, G. P. Wiederrecht, D. J. Gosztola, V. A. Podolskiy, and A. V. Zayats, Nat. Nanotechnol. 6, 107 (2011).

${ }^{20}$ J. B. Pendry, A. J. Holden, D. J. Robbins, and W. J. Stewart, IEEE Trans. Microwave Theory Tech. 47, 2075 (1999).

${ }^{21}$ E. Kim, F. Wang, W. Wu, Z. N. Yu, and Y. R. Shen, Phys. Rev. B 78, 113102 (2008).

${ }^{22}$ A. K. Sarychev and G. Tartakovsky, Phys. Rev. B 75, 085436 (2007).

${ }^{23}$ Z. H. Zhu, H. Liu, S. M. Wang, T. Li, J. X. Cao, W. M. Ye, X. D. Yuan, and S. N. Zhu, Appl. Phys. Lett. 94, 103106 (2009).

${ }^{24}$ N. I. Zheludev, S. L. Prosvirnin, N. Papasimakis, and V. A. Fedotov, Nat. Photon. 2, 351 (2008).

${ }^{25}$ B. Luk'yanchuk, N. I. Zheludev, S. A. Maier, N. J. Halas, P. Nordlander, H. Giessen, and C. T. Chong, Nat. Mater. 9, 707 (2010)

${ }^{26}$ S. M. Wang, Z. H. Zhu, J. X. Cao, T. Li, H. Liu, S. N. Zhu, and X. Zhang, Appl. Phys. Lett. 96, 113103 (2010).

${ }^{27}$ A. E. Miroshnichenko, S. Flach, and Y. S. Kivshar, Rev. Mod. Phys. 82, 2257 (2010).
${ }^{28}$ F. Hao, Y. Sonnefraud, P. Van Dorpe, S. A. Maier, N. J. Halas, and P. Nordlander, Nano Lett. 8, 3983 (2008).

${ }^{29}$ N. Verellen, Y. Sonnefraud, H. Sobhani, F. Hao, V. V. Moshchalkov, P. Van Dorpe, P. Nordlander, and S. A. Maier, Nano Lett. 9, 1663 (2009).

${ }^{30}$ V. A. Fedotov, M. Rose, S. L. Prosvirnin, N. Papasimakis, and N. I. Zheludev, Phys. Rev. Lett. 99, 147401 (2007).

${ }^{31}$ N. Liu, L. Langguth, T. Weiss, J. Kastel, M. Fleischhauer, T. Pfau, and H. Giessen, Nat. Mater. 8, 758 (2009).

${ }^{32}$ N. Papasimakis and N. I. Zheludev, Opt. Photonics News 20, 22 (2009).

${ }^{33}$ J. A. Fan, C. H. Wu, K. Bao, J. M. Bao, R. Bardhan, N. J. Halas, V. N. Manoharan, P. Nordlander, G. Shvets, and F. Capasso, Science 328, 1135 (2010).

${ }^{34}$ K. M. Dani, Z. Ku, P. C. Upadhya, R. P. Prasankumar, S. R. J. Brueck, and A. J. Taylor, Nano Lett. 9, 3565 (2009).

${ }^{35}$ D. J. Cho, W. Wu, E. Ponizovskaya, P. Chaturvedi, A. M. Bratkovsky, S. Y. Wang, X. Zhang, F. Wang, and Y. R. Shen, Opt. Express 17, 17652 (2009).

${ }^{36}$ N. Liu, M. Mesch, T. Weiss, M. Hentschel, and H. Giessen, Nano Lett. 10, 2342 (2010).

${ }^{37}$ H. Liu, J. Ng, S. B. Wang, Z. F. Lin, Z. H. Hang, C. T. Chan, and S. N. Zhu, Phys. Rev. Lett. 106, 087401 (2011).

${ }^{38}$ G. X. Li, Z. L. Wang, S. M. Chen, and K. W. Cheah, Opt. Express 19, 6348 (2011).

${ }^{39}$ H. Raether, Surface Plasmons on Smooth and Rough Surfaces and on Gratings (Springer Verlag, Berlin, 1988), p. 122.

${ }^{40}$ D. J. Griffiths, Introduction to Electrodynamics, $3^{\text {rd }}$ ed. (PrenticeHall International, New Jersey, 1999), p. 401.

${ }^{41}$ Y. R. Shen, The Principles of Nonlinear Optics (Willey, New York, 1984), p. 93.

${ }^{42}$ T. Utikal, T. Zentgraf, T. Paul, C. Rockstuhl, F. Lederer, M. Lippitz, and H. Giessen, Phys. Rev. Lett. 106, 133901 (2011). 